\title{
Dexmedetomidina, uma nova opção na anestesiologia veterinária
}

\section{Dexmedetomidine, a new option in veterinary anesthesia}

\author{
Caroline Floreoto Baldo ${ }^{1}$; Newton Nunes ${ }^{2 *}$
}

\begin{abstract}
Resumo
Com este trabalho, os autores procuram fornecer informações sobre o uso da dexmedetomidina, um novo agonista dos receptores adrenérgicos do tipo $\alpha-2$, na prática anestésica. São tecidas considerações relativas ao emprego e a farmacologia desse novo agente, suas vantagens e restrições de uso, bem como a avaliação quanto à sua eficiência e segurança quando utilizado em associação a outros agentes anestésicos. Complementarmente, procurou-se atualizar com literatura recente os conhecimentos dos profissionais que militam na área de Anestesiologia Veterinária.
\end{abstract}

Palavras-chave: Dexmedetomidine, anestesiologia veterinária.

\begin{abstract}
The aim of this work was to provide information about the use of dexmedetomidine, a new $\alpha$ - 2 agonist, in anesthetic practice. They comment about the pharmacology, its advantages and disadvantages, its efficiency and safety when associated with others anesthetic agents. Complementary, it was aimed to update the professionals that work with Veterinary Anesthesiology, through new papers and reports.

Key words: Dexmedetomidine, veterinary anesthesiology.
\end{abstract}

\section{Introdução}

No início dos anos 60, os agonistas dos receptores adrenérgicos do tipo $\alpha-2$ foram sintetizados para atuarem como descongestionante nasal. Esperavase que o uso de um agente vasoconstritor local promovesse a diminuição do edema da mucosa nasal desobstruindo a passagem de ar. Uma amostra do novo composto, atualmente conhecido como clonidina, foi encaminhada a um departamento médico para testar essa propriedade em humanos. Para tal, instilou-se algumas gotas nas narinas de uma secretária portadora de um resfriado e, para surpresa de todos, ela adormeceu e assim permaneceu por 24 horas (STAHLE, 1982). Ainda, desenvolveu hipotensão, bradicardia severa e apresentou xerostomia. Testes subseqüentes provaram a ação anti-hipertensiva da clonidina que foi introduzida no mercado em 1966. Desde então, essa classe de fármacos vem sendo empregada no tratamento de pacientes com hipertensão e naqueles viciados em drogas e álcool. Hoje, suas aplicações terapêuticas expandiram-se a fim de promover efeitos simpatolíticos, sedativos, hipnóticos, analgésicos, miorrelaxantes e ansiolíticos (KAMIBAYASHI; MAZE., 2000; CORTOPASSI; FANTONI, 2002).

O desenvolvimento de agonistas dos receptores adrenérgicos do tipo $\alpha-2$ altamente seletivos e específicos, despertaram na medicina humana o

\footnotetext{
1 Mestranda do Programa de Pós-graduação em Cirurgia Veterinária /FCAV/UNESP/Campus de Jaboticabal-SP.

2 Professor Assistente Doutor, Departamento de Clínica e Cirurgia Veterinária/FCAV/UNESP/Campus de Jaboticabal-SP. CEP: 14884-900, Jaboticabal, SP. E-mail: newton@ fcav.unesp.br

* Autor para correspondência.
} 
interesse pela sua reintrodução na prática anestésica. No final de 1999, nos EUA, o Food and Drug Administration (FDA) aprovou o uso da Dexmedetomidina (Tabela 1) para fins sedativos e analgésicos em pacientes do Centro de Terapia
Intensiva (CTI) (SCHOLZ; TONNER, 2000). Recentemente, foi lançada no mercado brasileiro, onde já iniciaram estudos sobre a eficácia desse fármaco em cães e gatos.

Tabela 1. Características e propriedades da dexmedetomidina, em humanos

\section{INDICAÇÃOO}

Sedação em pacientes na Unidade de Terapia Intensiva

\section{MECANISMO DE AÇÃO}

$\alpha 2$-agonista adrenérgico

\section{DOSAGEM E VIA DE ADMINISTRAÇÃO}

\begin{tabular}{ll}
\hline Dosagens usuais na rotina clínica & $1,0 \mu \mathrm{g} / \mathrm{kg}$ por 10 mins, depois $0,2 \mathrm{a} 0,7 \mu \mathrm{g} / \mathrm{kg}$ via IV \\
\hline \multicolumn{1}{c}{ PERFIL FARMACOCINÉTICO (concentração plasmática desejada de $0,6 \mu \mathrm{g} / \mathrm{L}$ ) } \\
\hline Área sob a curva concentração plasmática x tempo & $5,1 \mu \mathrm{g} / \mathrm{L} \mathrm{x} \mathrm{h}$ \\
Volume de distribuição & $1,33 \mathrm{~L} / \mathrm{kg}$ \\
Clearance & $0,495 \mathrm{~L} / \mathrm{h} \mathrm{x} \mathrm{kg}$ \\
Meia vida de distribuição & $6 \mathrm{~min}$. \\
Meia vida de eliminação & 2 horas \\
Via de eliminação & $95 \%$ renal (excretado como conjugados do ácido \\
& glicurônico) \\
Efeitos Adversos & Hipotensão, bradicardia e náusea \\
\hline
\end{tabular}

Fonte: Adaptado de Bhana, Goa e Mcclellan (2000)

\section{Farmacologia}

Os agonistas dos receptores adrenérgicos do tipo $\alpha-2$ possuem farmacologia bastante complexa, diferindo da maioria dos outros fármacos de uso rotineiro, por apresentarem potentes efeitos clínicos agindo especificamente em um único tipo de receptor (Figura 1). Esses receptores, com localização pré e pós-sináptica, possuem 4 sub-tipos $\left(\alpha-2_{\mathrm{A}}, \alpha-2_{\mathrm{B}}, \alpha-2_{\mathrm{C}} \mathrm{e}\right.$ $\left.\alpha-2_{\mathrm{D}}\right)$, que sabidamente são responsáveis por alguns efeitos específicos, a exceção do $\alpha-2_{D}$. O subtipo $\alpha-2_{\mathrm{A}}$ é responsável pela analgesia, anestesia e sedação; o subtipo $\alpha-2_{\text {В }}$ media as alterações cardiovasculares e o subtipo a- ${ }_{\mathrm{C}}$ promove os efeitos ansiolíticos (SCHOLZ; TONNER, 2000; SCHEININ et al., 1998). A elucidação da especificidade e localização desses subtipos de receptores, na opinião de Maze e Fujinaga (2000), é tão complexa quanto as bases moleculares do comportamento humano.

O mecanismo de ação básico dos agonistas dos receptores adrenérgicos do tipo $\alpha-2$ é caracterizado por diminuir a liberação de noradrenalina (NA) central e perifericamente, diminuindo a concentração de catecolaminas circulantes (em até 90\%) e atenuando a excitação do sistema nervoso central (SNC), especialmente na região conhecida por locus coeruleus (CHIU et al., 1995). Nesse local, os efeitos são controlados por receptores $\alpha-2_{\text {A }}$ (CALLADO; STAMFORD, 1999). 
A dexmedetomidina é um D-isômero com alta especificidade por receptores $\alpha-2$ (10 vezes mais, quando comparada à xilazina), que somada à levomedetomidina, o L-isômero, produz a mistura racêmica conhecida por medetomidina, descrita há 16 anos (SAVOLA et al., 1986 apud CULLEN, 1996). A levomedetomidina, considerada um ingrediente inativo (AANTAA; VIRTANEN; KALLIO, 1993), antagoniza as respostas hipnóticas do seu isômero, ligando-se a receptores $\alpha-1$ (KUUSELA et al., 2001a).

O mecanismo de ação da dexmedetomidina dáse pelo acoplamento aos receptores $\alpha-2$, principalmente pós-sinápticos e respectiva ativação das proteínas-G (proteínas ligadas à guanina) que agem diretamente nos canais iônicos de potássio aumentando sua condutância e hiperpolarizando a célula nervosa (BHANA; GOA; MCCLELLAN, 2000; SCHOLZ; TONNER, 2000).
Esse fármaco possui grande afinidade pelas proteínas plasmáticas (94\%), e após sofrer extensivo metabolismo hepático, é eliminado como um conjugado do ácido glicurônico pelos rins (95\%) não apresentando vestígios plasmáticos 10 horas após sua administração inicial por via intravenosa. Sua meia vida de eliminação, determinada em homens sadios, é de aproximadamente 2,3 horas (AANTAA et al., 1990). A sua farmacocinética foi marcadamente afetada em pacientes com insuficiência hepática dobrando-se o tempo para sua eliminação (BHANA; GOA; MCCLELLAN, 2000). Segundo relatos dos mesmos autores, a dexmedetomidina inibe o sistema enzimático do citocromo $\mathrm{P} 450$ in vitro, de modo reversível, demonstrando pouco potencial desta em interagir com outros fármacos metabolisados por tal via enzimática.

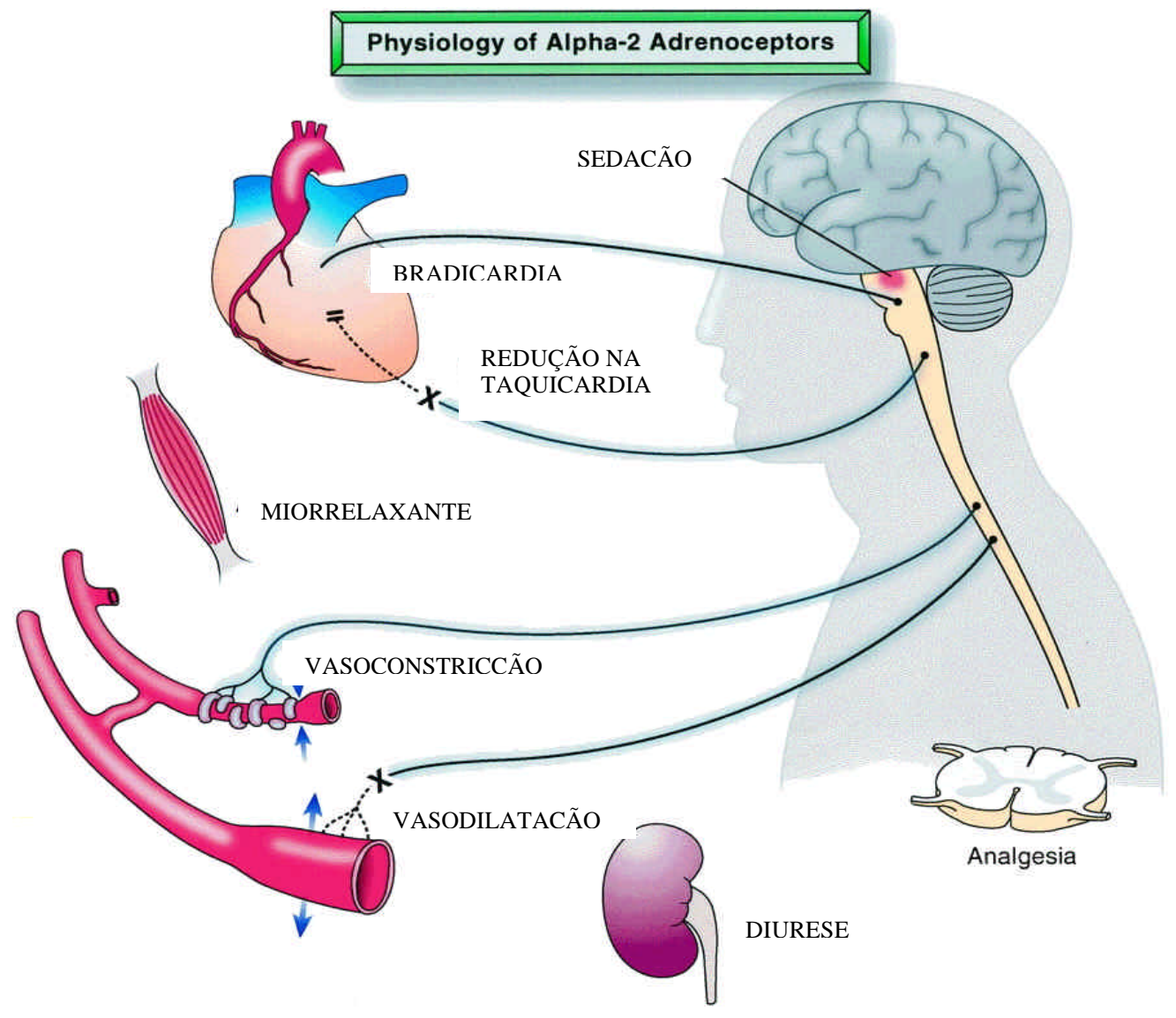

Figura 1. Respostas mediadas por receptores $\alpha-2$ adrenérgicos Fonte: Adaptado de Kamibayashi e Maze (2000, p.1345) 


\section{Efeitos Sedativos}

Como já citado, efeitos sedativos da dexmedetomidina são mediados centralmente no locus coeruleus onde concentram-se uma grande quantidade de receptores do tipo $\alpha-2$ adrenérgicos. Sendo assim, os nervos presentes nessa região, responsáveis pela transmissão de estímulos ao córtex cerebral e sistema límbico, tornam-se hiperpolarizados inibindo o impulso e conseqüentemente produzindo sedação (CULLEN, 1996).

Os efeitos, tanto desejáveis quanto adversos, são dependentes da dose (CORTOPASSI; FANTONI, 2002). Assim, doses maiores de dexmedetomidina produzem sedação mais profunda e prolongada, porém com efeitos adversos mais pronunciados.

Observou-se que a administração de $10 \mu \mathrm{g} / \mathrm{kg}$ por via intravenosa, promoveu decúbito lateral e esternal em cães, com picos de sedação e analgesia entre 10 e 20 minutos (KAMIBAYASHI; MAZE, 2000). Kuusela et al. (2001b) relataram que com o uso da dose de $20 \mu \mathrm{g} / \mathrm{kg}$ ocorreu profunda sedação e analgesia; com $2 \mu \mathrm{g} / \mathrm{kg}$ a sedação mostrou-se moderada e finalmente, $0,2 \mu \mathrm{g} / \mathrm{kg}$ promoveram sedação leve e nenhuma analgesia. O estudo de Hall et al. (2000) em humanos, demonstrou que durante a infusão contínua de duas doses diferentes, 0,2 e 0,6 $\mu \mathrm{g} / \mathrm{kg} / \mathrm{min}$., após bolus de $6 \mu \mathrm{g} / \mathrm{kg}$, não houve diferença na sedação registrado pelo monitor de índice biespectral (BIS). Ainda, os valores de BIS registrados apresentaram mínimos de 37 e 49, com média de 66, contudo, os pacientes eram facilmente acordados pela estimulação auditiva.

A sedação promovida pela dexmedetomidina raramente demanda o uso de medicação complementar para sua manutenção, como demonstrou um estudo realizado por Bhana, Goa e Mcclellan, Bhana (2000) que utilizaram pacientes humanos na Unidade de Terapia Intensiva (UTI). Somente $15 \%$ daqueles que receberam dexmedetomidina necessitaram de medicação complementar na manutenção da sedação com midazolam ou propofol, comparado a $60 \%$ do grupo de pacientes que receberam placebo.

\section{Efeito Analgésico}

A analgesia é produzida pela estimulação dos receptores espinhais e supra-espinhais, havendo evidências do envolvimento de mecanismos inibitórios pré e pós-sinápticos (PERTOVAARA et al., 1993, apud CULLEN, 1996). Radiologistas demonstraram alta concentração de receptores adrenérgicos do tipo $\alpha-2$ no corno dorsal da medula espinhal, onde as fibras nociceptivas possuem sinapses, e no tronco cerebral onde ocorre a modulação dos sinais álgicos (CULLEN, 1996). Estudos sugeriram o envolvimento de receptores pós-sinápticos mediando o possível efeito anestésico da dexmedetomidina em cães (VICKERY et al., 1988). Em modelos animais, verificou-se que não há um aparente efeito teto nos estudos realizados com a dexmedetomidina e o halotano, sugerindo que o agonista $\alpha-2$ possa ser um agente anestésico completo (KAMIBAYASHI; MAZE, 2000).

Bhana, Goa e Mcclellan (2000) observaram que em pacientes tratados com dexmedetomidina a dose de morfina para promover analgesia diminuiu 50\% em relação ao grupo tratado com placebo e aproximadamente $43 \%$ sequer necessitou de complementação.

\section{Efeitos Cardiovasculares}

A estimulação de diferentes regiões cerebrais responsáveis pelo controle autonômico aumenta o tônus vagal e inibe o tônus simpático. Receptores do tipo $\alpha-2$ adrenérgicos periféricos têm localização pré e pós-sináptica, sendo que a estimulação pré-sináptica inibe a liberação da noradrenalina promovendo bradicardia e hipotensão, enquanto que a estimulação pós-sináptica provoca hipertensão (CULLEN, 1996). Segundo Kamibayashi e Maze (2000), baixas doses de dexmedetomidina têm efeito predominantemente simpatolítico; doses altas ativam os receptores adrenérgicos do tipo $\alpha-2_{\mathrm{B}}$ na musculatura lisa dos vasos causando hipertensão. 
As variações hemodinâmicas coincidem com a redução plasmática de noradrenalina e adrenalina (BHANA; GOA; MCCLELLAN, 2000). O mesmo autor relata que a administração intravenosa de dexmedetomidina resulta em uma resposta bifásica da pressão arterial média (PAM), confirmando os achados de Kuusela et al. (2001b), traduzida por um aumento inicial, 5 a 10 minutos após sua administração, seguida de um decréscimo de aproximadamente 10 a 20\% dos valores basais. Já a freqüência cardíaca (FC) normalmente diminui devido a resposta de barorreflexo que promove vasoconstrição, para posterior estabilização deste parâmetro em valores superiores, porém, ainda aquém dos valores basais.

Aantaa et al. (1990), testaram quatro diferentes doses do fármaco $(0,167 ; 0,033 ; 0,67$ e $1 \mu \mathrm{g} / \mathrm{kg})$ e observaram efeitos não dependentes da dose como redução da pressão arterial sistólica e diastólica (PAS e PAD) 5 a 10 minutos após a administração inicial. A FC diminuiu $10 \%$ após administração das maiores doses. Em contraste, as duas menores doses promoveram um discreto aumento nesse parâmetro concordando com os achados de Kuusela et al. (2001b) em estudo utilizando cães.

Em outro estudo, observou-se que a administração de dexmedetomidina na dose de $10 \mu \mathrm{g} / \mathrm{kg}$ promoveu bradicardia, porém não demonstrou alterações na PAS, PAD e PAM o que poderia ser justificado pelos efeitos centrais e periféricos deste fármaco, produzindo ações contrárias de hipotensão e hipertensão, respectivamente (KITAHARA et al., 2002; KUUSELA et al., 2001a). Dependendo da dose, os efeitos periféricos podem mascarar os centrais promovendo estabilidade na pressão (PYPENDOP; VERSTEGEN, 1998).

Kitahara et al. (2002) ainda observaram redução no débito cardíaco (DC) e no índice cardíaco relacionados à bradicardia e aumento da resistência vascular periférica (RVP) atribuídos aos efeitos de vasoconstrição direta.
Mendes et al. (2002) em estudo realizado com gatos, observaram que a dexmedetomidina associada à quetamina, ao contrário da xilazina e romifidina, não induziu o aparecimento de bloqueios atrioventriculares (BAV), exercendo efeitos mais brandos sobre a condução e freqüência cardíaca. Tal achado opõe-se aos citados por Kuusela et al. (2001b) que, em cães, observaram freqüentes episódios de BAV de primeiro grau, e alguns de segundo grau, com acentuada arritmia sinusal.

Zornow et al. (1990 apud CULLEN, 1996), descreveram que o fluxo sangüíneo cerebral foi marcadamente reduzido após administração intravenosa de $10 \mu \mathrm{g} / \mathrm{kg}$ desse fármaco, sem contudo, afetar a demanda de oxigênio em cães anestesiados com isoflurano e mantidos sob ventilação controlada. Segundo Ohata et al. (1999), a administração sistêmica da dexmedetomidina atenua a vasodilatação cerebral induzida pelo isoflurano e sevoflurano. Tal efeito não se caracterizou como dependente da dose e não diferiu entre os anestésicos estudados.

\section{Efeitos Respiratórios}

A dexmedetomidina na dose de $2 \mu \mathrm{g} / \mathrm{kg}$ administrada por infusão contínua com duração de 2 minutos, produz discreto aumento na pressão parcial de dióxido de carbono e redução no volume minuto com pequena mudança na freqüência respiratória (BHANA; GOA; MCCLELLAN, 2000). Os mesmos autores relataram que a infusão contínua desse fármaco durante 24 horas, na tentativa de se obter a concentração plasmática de 0,3 a 1,25 $\mu \mathrm{g} / \mathrm{L}$ ), não causou depressão respiratória nos pacientes, mantendo a oximetria em valores acima de $90 \%$. Já, Hall et al. (2000), observaram valores acima de $95 \%$ e freqüência respiratória e concentração de $\mathrm{CO}_{2}$ ao final da expiração inalterados.

Kuusela et al. (2001b) observaram que em cães tratados com $20 \mu \mathrm{g} / \mathrm{kg}$ do fármaco, a pressão parcial de $\mathrm{O}_{2}$ diminuiu discretamente em relação às doses de 2 e $0,2 \mu \mathrm{g} / \mathrm{kg}$. Em todos os tratamentos não foi 
observado redução significativa na freqüência respiratória, contudo, houve acréscimo na pressão parcial de $\mathrm{CO}_{2}$ e redução significativa no $\mathrm{pH}$ arterial e na concentração de bicarbonato, principalmente nas doses de 2 e $20 \mu \mathrm{g} / \mathrm{kg}$ traduzindo um quadro de acidose metabólica.

\section{Outros Efeitos}

No sistema músculo-esquelético, os efeitos miorrelaxantes dos agonistas $\alpha 2$ aparentemente não são mediados na junção neuromuscular, mas sim centralmente, de maneira semelhante aos benzodiazepínicos. A dexmedetomidina produz flacidez muscular prevenindo a rigidez causada pelos opióides (SCHOLZ; TONNER, 2000).

No trato gastrointestinal, os efeitos incluem a inibição da secreção gástrica, pela ativação de adrenoceptores $\alpha 2$ centrais e periféricos, e da motilidade intestinal em cães sob jejum ou não (CULLEN, 1996). A dexmedetomidina, administrada através de infusão contínua, pode causar náusea em pacientes humanos (BHANA; GOA; MCCLELLAN, 2000). Nos animais, supõe-se que tal fármaco promova o vômito agindo na zona cerebral correspondente, cujo efeito já é conhecido para a xilazina (COLBY et al., 1981, apud CULLEN, 1996).

Relativo a função endócrina, os agonistas dos receptores adrenérgicos do tipo $\alpha-2$, potencializando a liberação do hormônio de crescimento, efeito este, improvável de apresentar consequiências clinicamente nocivas Hayashi e Maze (1993 apud CULLEN, 1996). Ainda, a glicemia aumenta após o uso dessa classe de fármacos, por estes agirem nos receptores $\alpha-2$ pós-sinápticos das células b do pâncreas diminuindo o nível de insulina circulante (THURMON; TRANQUILLI; BENSON, 1996; CULLEN, 1996). Os mesmos autores também relataram a diminuição da reabsorção de água nos néfrons, da liberação da vasopressina e do hormônio antidiurético $(\mathrm{ADH})$ pela pituitária, aumentando o débito urinário.
No sistema genital feminino, a motilidade uterina foi reduzida, como citado por Cullen (1996), após a administração de $20 \mu \mathrm{g} / \mathrm{kg}$ de medetomidina, porém, aumentada após uma dose de 40 e $60 \mu \mathrm{g} / \mathrm{kg}$. Em nenhum caso registrou-se a ocorrência de abortos.

A hipotermia, evento comum nos pacientes submetidos a sedação ou anestesia, é provocada por receptores presentes no hipotálamo que são deprimidos pelos agonistas $\alpha-2$ (CULLEN, 1996).

\section{Associações Farmacológicas}

As doses propostas de dexmedetomidina isolada ou associada a outros fármacos para pequenos animais foram de 2,10 e $20 \mu \mathrm{g} / \mathrm{kg}$ por via intravenosa ou intramuscular (KITAHARA et al., 2002; MENDES et al., 2002; KUUSELA et al., 2001a; KUUSELA et al., 2001b).

Desde meados dos anos 80, muitos estudos comprovaram a redução da concentração alveolar mínima (CAM) dos anestésicos inalatórios proporcionada pelos agonistas dos receptores adrenérgicos do tipo $\alpha-2$. Scholz e Tonner (2000), relataram redução na CAM do halotano em mais de 90\%. Contudo, o mesmo autor cita que para o sevoflurano a redução foi de apenas $17 \%$ nos pacientes de 55 a 70 anos. A redução na CAM do isoflurano foi de $40 \%$ após administração de $2 \mu \mathrm{g} / \mathrm{kg}$ do agonista de receptores adrenérgicos do tipo $\alpha-2$ e de $85 \%$ após administração de 20 (KUUSELA et al., 2001b). Os mesmos autores referem que nas doses de dexmedetomidina anteriormente citadas (2 e $20 \mu \mathrm{g} / \mathrm{kg}$ ), reduzidas doses de propofol, 2,7 e 0,8 $\mu \mathrm{g} / \mathrm{kg}$ respectivamente, foram necessárias para indução, observando-se episódios de apnéia transitória, vômito e dispnéia. Na recuperação, doses mais altas resultaram em tempos de extubação, decúbito lateral e esternal e deambulação maiores.Constatou-se uma redução na dose de indução e manutenção do tiopental em 30\% (AANTAA et al., 1990). No estudo de Mendes et al. (2002), a bradicardia promovida por $10 \mu \mathrm{g} / \mathrm{kg}$ de 
dexmedetomidina não foi significativamente revertida com $5 \mu \mathrm{g} / \mathrm{kg}$ de cetamina.

\section{Antagonista: Atipamezole}

O uso de antagonistas específicos dos receptores adrenérgicos do tipo $\alpha-2$, possibilita o controle dos efeitos dos agonistas, sendo estes produzidos ou revertidos à semelhança dos relaxantes musculares, o que se faz de grande interesse para a Anestesiologia (KAMIBAYASHI; MAZE, 2000).

Atipamezole, o mais recente antagonista, foi descrito por Scheinin et al. (1998), por promover reversão dos efeitos sedativos e simpatolíticos de maneira dose dependente, em voluntários que receberam dexmedetomidina. Possui meia vida de eliminação de 2 horas, coincidindo com o da dexmedetomidina, sendo improvável a recorrência dos efeitos uma vez antagonizados (KAMIBAYASHI; MAZE, 2000). A sua seletividade por receptores adrenérgicos do tipo $\alpha-2$ é de 200 a 300 vezes maior que a ioimbina com uma afinidade 100 vezes maior (CULLEN, 1996). Segundo o mesmo autor, a reversão dos efeitos, em cães, ocorre com uma dose 4 a 6 vezes maior que àquela administrada de medetomidina e, em gatos, 2 a 4 vezes mais. A supressão dos efeitos começa em cinco minutos, apresentando em cães, poucos efeitos adversos como micção, salivação e hipotensão. Em gatos, não foram observados a ocorrência desses efeitos.

\section{Conclusão}

O emprego da dexmedetomidina promete benefícios na Anestesiologia clínica pelo aspecto farmacológico e pela alta especificidade que oferece. Contudo, suas vantagens e desvantagens devem ser melhor elucidadas por meio de estudos clínicos tanto na Medicina Humana quanto na Veterinária.

Certamente, terá grande valor clínico pelo fato não somente promover sedação e analgesia, mas também possivelmente anestesia, ainda com a vantagem de poder ter seus efeitos antagonizados ao final dos procedimentos, o que certamente fará parte do futuro da Anestesiologia.

\section{Referências}

AANTAA, R. E.; KANTO, J. H.; SCHEININ, M.; KALLIO, A. M. I.; SHEININ, H. Dexmedetomidine, an alpha 2 adrenoceptor agonist, reduces anesthetic requirements for pacients undergoing minor Gynecologic Surgery. Anesthesiology, Philadelphia, v.73, n.2, p.230-235, 1990.

AANTAA, R. E; VIRTANEN, R.; KALLIO, A. M. I. Dexmedetomidine, a novel $\alpha 2$ adrenergic agonist: a review of its pharmacodynamic characteristics. Drugs of the Future, Barcelona, v.18, p.49-56, 1993.

BHANA, N.;GOA, K.; MCCLELLAN, K.J. Dexmedetomidine. Drugs, Sydney, v.59, n.2, p.263-268, 2000.

CALLADO, L. F.; STAMFORD, J. A. Alpha 2 but not alpha $2 \mathrm{~B} / \mathrm{C}$ adrenoceptors modulate noradrenaline release in rat locus coeruleus: voltammetric data. European Journal of Pharmacology, Amsterdan, v.366, n.1, p.3539, 1999.

CHIU, T. H.; CHEN, M. J.; YANG, J. J.; TANG, F. I. Action of dexmedetomidine on rat locus coeruleus neurones: intracellular recording in vitro. European Journal of Pharmacology, Amsterdan, v.285, n.3, p.261-268, 1995.

CORTOPASSI, S. R. G.; FANTONI, D. T. Medicação Préanestésica. In: _. Anestesia em cães e gatos. São Paulo: Roca, 2002. p.151-158.

CULLEN, L. K. Medetomidine sedation in dogs and cats: A review of its pharmacology, antagonism and dose. British Veterinary Journal, London, v.152, n.5, p.519-531, 1996.

HALL, J.E.; UHRICH, T. D.; BARNEY, J. A.; ARAIN, S. R.; EBERT, T. J. Sedative, Amnesic, and Analgesic properties of small dose dexmedetomidine infusions. Anesthesia \& Analgesia, Baltimore, v.90, n.3, p.699-705, 2000.

KAMIBAYASHI, T.; MAZE, M. Clinical Uses of a2 adrenergic agonists. Anesthesiology, Philadelphia, v.93, n.5, p.1345-1349, 2000.

KITAHARA, F. R.; CORTOPASSI, S. R. G.; OTSUKI, D. A.; INTELIZANO, T. R.; FANTONI, D. T.; AULER JR, J. O.C. Efeitos hemodinâmicos da dexmedetomidina em cães. Estudo experimental. Revista Brasileira de Ciências Veterianrias, Niterói, v.9, n.1, p.128-130, 2002.

KUUSELA, E.; RAEKALLIO, M.; VÄISÄNEN, M.; MYKKANEN, K.; ROPPONEN, H.; VAINIO, O. Comparison of dexmedetomidine as premedicants in dogs undergoing propofol-isoflurane anesthesia. American 
Journal of Veterinary Research, Schaumburg, v.62, n.7, p.1073-1079, 2001b.

KUUSELA, E.; VAINIO, O.; KAISTINEN, A.; KOBYLIN, $\mathrm{S}$; RAEKALLIO, M. Sedative, analgesic, and cardiovascular effects of levomedetomidine alone and in combimation with levomedetomidine in dogs. American Journal of Veterinary Research, Schaumburg, v.62, n.4, p.616-621, 2001a.

MAZE, M.; FUJINAGA, M. Alpha 2 adrenoceptors in pain modulation. Which subtype should be targeted to produce analgesia? Anesthesiology, Philadelphia, v.92, n.4, p.934-936, 2000.

MENDES, G. M.; SILVA, C.E. V.; SELMI, A. L.; BARBUDOSELMI, G. R.; LINS, B. T.; FIGUEIREDO, J. B.; MCMANAUS, C. Alterações eletrocardiográficas da dexmedetomidina, romifidina ou xilazina em associação à cetamina em gatos. Revista Brasileira de Ciências Veterinárias, Niterói, v.9, n.1, p.137-139, 2002.

OHATA, H.; IIDA, H.; DOHI, S.; WATANABE, Y. Intravenous dexmedetomidine inhibits cerebrovascular dilation induced by isoflurane and sevoflurane in dos. Anesthesia \& Analgesia, Baltimore, v.89, n.2, p.370-377, 1999.
PYPENDOP, B. H.; VERSTEGEN, J. P. Hemodynamic Effects of medetomidine in the dog: a dose tritration Study. Veterinary Surgery, Philadelphia, v.27, n.6, p.612-622, 1998.

SCHEININ, M.; SALLINEN, J.; HAAPALINNA, A.; VIITAMAA, T.; KOBILKA, B. K. Adrenergic $\alpha 2_{\text {c }}$ receptors modulate the acoustic startle reflex, prepulse inhibition and aggression in mice. Journal of Neurological Science, Amsterdan, v.18, n.10, p.3035-3042, 1998.

SCHOLZ, J.; TONNER, P. H. Alpha 2 adrenoceptor agonists in anaesthesia: a new paradigm. Current Opinion in Anaesthesiology, London, v.13, n.4, p.437-442, 2000.

STAHLE, H. Clonidine. In: BINDRA, J. S.; LEDNICER, D. Chronicles of drug discovery. New York: Wiley, 1982. v.1, p.87-111.

THURMON, J. C.; TRANQUILLI, W. J.; BENSON, G. J. Section I :Overview of Veterinary Anethesia. In: _Lumb \& Jone's Veterinary Anesthesia. 3.ed. Baltimore, Maryland: William \& Wilkins, 1996. p.1-60.

VICKERY, R. G.; SHERIDAN, B. C.; SEGAL, I. S.; MAZE, M. Anesthetic and hemodynamic effects of the stereoisomers of medetomidine an 2 adrenergic agonist, in halotane anesthetized dogs. Anesthesia \& Analgesia, Baltimore, v.67, n.7, p.611-615, 1988. 